\title{
Encefalitis herpética. Serie clínica de 15 casos confirmados por reacción de polimerasa en cadena
}

\author{
ALBERTO FICA C., CARLOS PÉREZ C., PAULA REYES O.*, \\ SANDRA GALLARDO P.*, XIMENA CALVO P*. y ANA M. SALINAS S.
}

\section{Herpetic encephalitis. Case series of 15 patients confirmed by polymerase chain reaction}

Encephalitis by herpes simplex virus (HSV) is an sporadic and the most important cause of encephalitis in the western world. The aim of this study was to describe the main clinical features and response to therapy in a representative serie of cases. Fifteen cases confirmed by polymerase chain reaction were identified in two universitary hospitals in Santiago. Average age was 41 years (range 578 ) being $80 \%$ over 30 years old. Most cases presented with fever and sensorial involvement (80\%) or headache $(67 \%)$ and only a minority with seizures or focal signs $(\leq 15 \%)$. Extracerebral herpetic lesions were present in two patients (13\%). Average length of symptoms was 3. 8 days and most cases were associated to type $1 \mathrm{HSV}(86.7 \%)$. Changes were detected in $91.7 \%$ of those evaluated with electroencephalogram, in $81.8 \%$ of those evaluated with nuclear magnetic resonance and in only $13.3 \%$ of those evaluated with a cerebral CT-scan. All patients were treated with acyclovir and casefatality ratio was $13.3 \%$, although one death in a patient with AIDS and CNS lymphoma could not be related to HSV. Six patients $(40 \%)$ showed neurological deficit at discharge. Death or neurological deficit at discharge was associated with a delay $>3$ days before acyclovir therapy. ( $p=0.01$, twotailed Fisher test)

Key words: Herpetic encephalitis, polymerase chain reaction, Central nervous system infection.

Palabras clave: Encefalitis herpética, reacción de polimerasa en cadena, infección del sistema nervioso central.

\section{Introducción}

La encefalitis herpética es una enfermedad grave asociada al virus herpes simplex 1 ó 2 (VHS 1 o VHS 2). Aparece en forma endémica, sin estacionalidad y en pacientes inmunocompetentes. Puede manifestarse como un cuadro clásico de encefalitis infecciosa con fiebre, cefalea y alteración en el nivel de conciencia. Debido al compromiso del lóbulo temporal, las manifestaciones clínicas pueden incluir también alucinaciones, afasia y cambios de personalidad. La mayor parte de los casos en adultos se presenta en personas sobre 35 años de edad y su letalidad es $>70 \%$ sin tratamiento. Las secuelas en los pacientes tratados son significativas ${ }^{1}$.

El diagnóstico de la encefalitis herpética ha cambiado en los últimos años gracias al advenimiento de la RPC en LCR para VHS, una estrategia rápida y de alta sensibilidad y especificidad, que en nuestro medio ha permitido reemplazar los diagnósticos presuntivos por TAC o electroencefalograma (EEG) o los bajos rendimientos del aislamiento viral en el LCR.

\footnotetext{
Hospital Clínico Universidad de Chile. Santiago, Chile:

Sección Infectología, (AFC).

Estudiantes de Medicina (PRO, SGP, XCP).

Programa de Enfermedades Infecciosas, Departamento de Medicina Hospital Clínico.

Pontificia Universidad Católica de Chile (CPC).

Laboratorio Clínico (AMSS).
} 
El tratamiento de esta condición también ha experimentado importantes modificaciones en las dos últimas décadas gracias a la incorporación de aciclovir, el que ha permitido mejorar significativamente el pronóstico de esta enfermedad ${ }^{2}$.

A pesar de que existen publicaciones y trabajos de investigación presentados en diferentes congresos en nuestro medio ${ }^{3-7}$, una revisión clínica de esta patología se hace necesaria debido al reducido tamaño de cada una de las series clínicas publicadas hasta ahora en Chile (uno a 5 casos por reporte), a la antiguiedad de estas publicaciones (sólo una en los últimos 10 años) y a los cambios en las prácticas diagnósticas y terapéuticas evidenciadas en las últimas décadas. El uso de aciclovir se hizo extensivo sólo en los años 90 después de aparecida la mayor parte de las publicaciones sobre el tema en Chile. Por ejemplo, sólo 2 de 5 pacientes comunicados por Wu et al, entre 1980 y 1984 fueron tratados con medicamentos antivirales ${ }^{6}$. En contraste, todos los casos reportados por Schultz et al fueron tratados con aciclovir a comienzos de los $90^{5}$.

La siguiente recopilación tiene como objetivos describir las principales manifestaciones clínicas de esta enfermedad en una serie mayor de pacientes, evaluar la respuesta terapéutica a aciclovir, sistematizar la evolución clínica de estos cuadros, medir la concordancia entre la información obtenida por diferentes estrategias de apoyo diagnóstico y finalmente, comparar nuestros resultados con aquellos reportados en la literatura.

\section{Pacientes y métodos}

Para ampliar el número de pacientes se incluyó la experiencia clínica de dos hospitales universitarios. Para optimizar el análisis de los resultados y evitar sesgos de inclusión, sólo se incluyeron pacientes con un diagnóstico confirmado por RPC para VHS 1 o VHS 2 en el LCR, ya que esta estrategia representa el método de elección actual. No se incluyeron casos confirmados por aislamiento viral, síntesis de anticuerpos específicos en el LCR o biopsia cerebral, estas técnicas no se aplican o no se aplicaron rutinariamente en Chile para este propósito y además, ningún caso fue confirmado de esta manera al hacer una búsqueda por diagnóstico de egreso en uno de los hospitales.

En el Hospital Clínico de la Universidad de Chile (HCUCH) se utilizó una pesquisa mediante diagnóstico de egreso en una ventana amplia de tiempo (13 años) con búsqueda de resultados de laboratorio y su correspondiente confirmación por el Laboratorio de Virología de la Facultad de Medicina de la misma Universidad, o por otros centros (BIOS-Chile). Los diagnósticos de egreso incluyeron los códigos 0543 (encefalitis herpética) desde el año 1991 hasta el 2001 y luego el código B00.4 (encefalitis, meningoencefalitis herpética) hasta abril del 2004, según la Clasificación Estadística Internacional de Enfermedades y Problemas relacionados con la Salud. (CIE 10, décima revisión. OPS Publicación científica n ${ }^{\circ} 554$, vol 3 1995).

En el Hospital Clínico de la Pontificia Universidad Católica (HCPUC) se efectuó una búsqueda de todas las RPC positivas para VHS 1 o VHS 2 en LCR efectuadas por el Laboratorio de Microbiología y que contaran con información clínica disponible.

La RPC se efectuó en ambos centros con protocolos diferentes. En el Laboratorio de Virología perteneciente a la Universidad de Chile se utilizaron dos metodologías durante el período, inicialmente la técnica descrita por Schlesinger et $\mathrm{al}^{8}$ y luego una RPC multiplex para herpesvirus descrita por Casas et $\mathrm{al}^{9}$. La detección de ADN de herpesvirus para las muestras estudiadas en la Pontificia Universidad Católica se realizó para todos los casos, exceptuando uno, mediante un método comercial (Herplex, PharmaGen $\left.{ }^{\circledR}\right)$ tipo ELISA con hibridación con sondas para la identificación del producto y la diferenciación entre VHS 1 y VHS 2. En la muestra restante se desarrolló con RPC en tiempo real en un equipo Light-Cycler ${ }^{\circledR}$ (Roche) de acuerdo a las recomendaciones del fabricante.

Los casos reclutados fueron analizados mediante una ficha estructurada que registró los datos demográficos, manifestaciones clínicas, resultados de diagnóstico de apoyo incluyendo características del estudio del LCR, inicio del tratamiento y respuesta clínica.

Las comparaciones estadísticas fueron efectuadas mediante la prueba de Fisher bilateral.

\section{Resultados}

Identificación de casos. Un total de 15 casos en 15 pacientes fue identificado en ambos hospitales universitarios. Cuatro en el HCUCH y 11 en el HCPUC. Otros 3 casos adicionales en el HCPUC no pudieron ser analizados porque las fichas estaban extraviadas. De la misma manera, un caso evaluado en el HCUCH no pudo ser incluido, a pesar de estar confirmado por RPC, debido a que no se encontró ningún documento oficial que lo ratificara y no pudo ser rastreado en ningún laboratorio. 
Distribución estacional, anual y etaria de los casos. La mayor incidencia de casos fue observada en el mes de mayo con 5 eventos (un tercio del total). El resto de los casos se distribuyó en forma uniforme durante el resto del año. El primer caso se identificó durante el año 1998, luego la cifra se incrementó a 4 casos por año desde 1999 hasta el 2001. La cifra se redujo el año 2002 a 2 eventos sin identificar nuevos casos el año 2003. El 80\% de los pacientes afectados tenía 30 o más años (Tabla 1).

Manifestaciones clínicas al ingreso y alteraciones en el LCR. Las principales manifestaciones clínicas correspondieron a fiebre, cefalea y compromiso de conciencia y, con menor frecuencia, focalización o convulsiones. (Tabla 1) Otros síntomas infrecuentes incluyeron vómitos, afasia, alucinaciones, dolor abdominal o mutis- mo. Dos pacientes presentaron otra localización de la infección herpética $(13,4 \%)$, un caso con estomatitis y otro con vulvovaginitis. La duración de los síntomas antes del ingreso fue 3,8 días promedio y en ningún caso superior a 10 días. Sólo un bajo número de pacientes fue evaluado mediante la escala Glasgow, lo que impidió evaluar este parámetro. La VHS presentó una elevación moderada y estaba en rangos normales en un tercio de los pacientes. Las alteraciones pesquisadas en el LCR correspondieron a pleocitosis (valor promedio $804 / \mathrm{mm}^{3}$ ) de predominio mononuclear, euglucorraquia y discreto aumento de las proteínas (valor promedio 118,1 $\mathrm{mg} / \mathrm{dL}$ ). Con la excepción de un paciente con linfoma del SNC que presentó hipoglucorraquia extrema $(2 \mathrm{mg} / \mathrm{dL})$, todos los otros casos presentaron una cifra $\geq 40 \mathrm{mg} / \mathrm{dL}$. Tres pacientes no presentaron pleocitosis (20\%), y el rango de

Tabla 1. Distribución de diferentes parámetros clínicos y de laboratorio en 15 pacientes con encefalitis herpética

\begin{tabular}{|c|c|c|}
\hline Parámetro & Frecuencia o valor promedio & Rango o porcentaje \\
\hline \multicolumn{3}{|l|}{ Datos demográficos } \\
\hline Distribución por sexo (M/F) & $10 / 5$ & $66 \% / 34 \%$ \\
\hline Edad (años) & 41 & $5-78$ \\
\hline Edad $\geq 30$ años & 12 & 80 \\
\hline Comorbilidad & 9 & $60 *$ \\
\hline \multicolumn{3}{|l|}{ Manifestaciones clínicas } \\
\hline Fiebre & 12 & 80 \\
\hline Cefalea & 10 & 66,6 \\
\hline Compromiso de conciencia & 12 & 80 \\
\hline Focalización & 2 & 13,3 \\
\hline Convulsiones & 3 & 15 \\
\hline Herpes labial o genital & 2 & 13,3 \\
\hline Duración síntomas (días) & 3,8 & $1-10$ \\
\hline \multicolumn{3}{|l|}{ Laboratorio } \\
\hline VHS > $20 \mathrm{~mm} / \mathrm{h}$ & 10 & 66,6 \\
\hline VHS mm/h & 40,8 & $8-140$ \\
\hline \multicolumn{3}{|l|}{ Valores en LCR } \\
\hline Glucosa mg/dL & 60,4 & $2-104$ \\
\hline Proteína mg/dL & 118,1 & $25-480$ \\
\hline Presencia de eritrocitos & & 60 \\
\hline Recuento de eritrocitos $/ \mathrm{mm}^{3}$ & 274,7 & $0-2.690$ \\
\hline Presencia de pleocitosis & & 80 \\
\hline Recuento de leucocitos $/ \mathrm{mm}^{3}$ & 804,6 & $2-8.640$ \\
\hline$\%$ mononucleares & 96,9 & $80-100$ \\
\hline \multicolumn{3}{|l|}{ Tipificación viral } \\
\hline VHS-1 / VHS-2 & $13 / 2$ & $86,7 / 13,3$ \\
\hline
\end{tabular}

*: Síndrome depresivo $(\mathrm{n}=2)$, obesidad, jaqueca clásica, hipotiroidismo, cirrosis hepática (Child A) de causa no precisada, infección VIH con linfoma del SNC, diabetes mellitus tipo 2 y retardo mental por hipoxia neonatal $(\mathrm{n}=1$ cada uno). 
células mononucleares fue de 80 a $100 \%$. El $60 \%$ de los pacientes presentó eritrocitos en el LCR. La mayor parte de los aislados virales correspondió a VHS-1. (Tabla 1) Sólo 2 casos estuvieron asociados a VHS-2 lo que no permitió hacer comparaciones sobre el cuadro clínico.

Estudio neurodiagnóstico. Doce pacientes tuvieron un estudio de EEG, 11 de ellos con un resultado alterado $(91,7 \%)$. (Tabla 2) La alteración más frecuente correspondió a actividad epileptiforme focal $(45,5 \%)$, secundada por focalización temporal lenta $(36,4 \%)$, "PLEDS" (periodic lateralizing epileptiform discharges) en 3 casos $(27,3 \%)$ y lentitud en el ritmo de base en 2 casos $(18,2 \%)$ (fenómenos no excluyentes) Exceptuando un caso, todos los pacientes con alteraciones en el EEG tuvieron manifestaciones temporales $(63,6 \%)$ o frontales aisladas $(27,3 \%)$ o frontotemporales $(9,1 \%)$. De los 11 pacientes estudiados con RM, 9 presentaron alteraciones. $(81,8 \%)$. Todos ellos mostraron compromiso frontal y/o temporal (datos no mostrados). Todos los pacientes fueron estudiados con TAC cerebral y sólo 2 de ellos tuvieron alteraciones al examen. (13,3\%) (Tabla 2) La TAC se efectuó antes de completar una semana de evolución en
$73 \%$ de los pacientes. En los 2 pacientes en los que el estudio estuvo alterado, este examen se efectuó a las 24 horas de evolución y a los 10 días, respectivamente. Los estudios tomográficos no fueron repetidos. El $90 \%$ de los estudios de $\mathrm{RM}$ fue realizado en la primera semana de evolución de los síntomas.

Tratamiento y evolución. Todos los pacientes en la serie fueron tratados con aciclovir endovenoso. La latencia para su inicio fue de 4,5 días promedio desde el inicio de los síntomas (rango 1 a 10 días) y 0,93 días desde el ingreso (rango 0 a 5 días). El $60 \%$ inició el tratamiento el mismo día de ingreso y $86,7 \%$ en las primeras 72 horas. Las dosis fueron apropiadas en todos ellos (datos no mostrados) al igual que la duración del tratamiento (promedio 14,1 días) (Tabla 2).

Dos pacientes fallecieron en la serie aunque la muerte en uno de ellos, afectado por SIDA y linfoma tipo Burkitt del SNC, no pudo ser atribuida directamente a la infección herpética. Se comprobó por citometría de flujo en una muestra de LCR el compromiso linfomatoso del SNC; la carga viral de VIH era de 300.000 copias/mL y el recuento CD4 de 300/ $\mathrm{mm}^{3}$. Este paciente recibió dos días de tratamiento con aciclovir antes de

Tabla 2. Resultados del estudio neurodiagnóstico, tratamiento y evolución en 15 casos de encefalitis herpética

\begin{tabular}{|c|c|c|}
\hline Parámetro & Frecuencia o valor promedio & Rango o porcentaje \\
\hline \multicolumn{3}{|l|}{ Estudio neurodiagnóstico } \\
\hline EEG alterado $\mathrm{n} /$ total \% & $11 / 12$ & $91,7 \%$ \\
\hline Actividad epileptiforme focal & $5 / 11$ & $45,5 \%$ \\
\hline Focalización lenta temporal & $4 / 11$ & $36,4 \%$ \\
\hline PLEDS* & $3 / 11$ & $27,3 \%$ \\
\hline Lentitud difusa del ritmo de base & $2 / 11$ & $18,2 \%$ \\
\hline Con alteraciones temporales $* *$ & $7 / 11$ & $63,6 \%$ \\
\hline Con alteraciones frontales $* *$ & $4 / 11$ & $36,4 \%$ \\
\hline TAC alterada $\mathrm{n} /$ total, $\%$ & $2 / 15$ & $13,3 \%$ \\
\hline RNM alterada $\mathrm{n} /$ total, $\%$ & $9 / 11$ & $81,8 \%$ \\
\hline \multicolumn{3}{|l|}{ Tratamiento } \\
\hline Tratamiento con aciclovir $\mathrm{n}, \%$ & 15 & $100 \%$ \\
\hline Latencia desde inicio cuadro & 4,5 días & 1 a 10 días \\
\hline Latencia desde ingreso & 0,93 días & 0 a 5 días \\
\hline Duración tratamiento & 14,1 días & 2 a 21 días*** \\
\hline \multicolumn{3}{|l|}{ Evolución } \\
\hline Duración hospitalización & 21,4 días & 5-64 días**** \\
\hline Fallecidos n, $\%$ & 2 & $13,3 \%$ \\
\hline Con secuelas al alta $\mathrm{n}, \%$ & 6 & $40 \%$ \\
\hline Sin alteraciones $\mathrm{n}, \%$ & 7 & $46,7 \%$ \\
\hline
\end{tabular}

*: Periodic lateralizing epileptiform discharges; **: Un paciente con lesiones frontotemporales; ***: La duración del tratamiento fue de 2 días en uno de los pacientes que falleció; **** Excluye un paciente trasladado a las 48 horas a otro centro para continuar tratamiento. 
Tabla 3. Secuelas o muerte al egreso según duración de la enfermedad antes del inicio del tratamiento en 14 pacientes*

\begin{tabular}{cccc}
\hline & \multicolumn{2}{c}{ Condición al egreso } \\
$\begin{array}{c}\text { Días de enfermedad } \\
\text { hasta inicio tratamiento }\end{array}$ & $\begin{array}{c}\text { Muerte o secuela } \\
\text { neurológica }\end{array}$ & Sin alteraciones & Total \\
\hline$\leq 3$ & 0 & 5 & 5 \\
$>3$ & 7 & 2 & 9 \\
Total & 7 & 7 & 14 \\
\hline
\end{tabular}

$\mathrm{p}=0,01$ por prueba bilateral de Fisher; * excluye un caso del cual no se tiene antecedentes sobre duración de los síntomas antes del ingreso.

fallecer. El otro paciente falleció por un coma atribuible a su encefalitis. La letalidad en esta serie alcanzó a 13,3\%. De los restantes pacientes, $6(40 \%)$ egresaron con alguna secuela que incluía disfasia, afasia o anomia, paresia facial o hemiparesia, alteraciones de memoria, epilepsia focal, depresión o dificultades en la capacidad de abstracción. Sólo 7 pacientes fueron dados de alta sin alteraciones evidentes (46,7\%) (Tabla 2).

Una mayor latencia hasta el inicio del tratamiento con aciclovir (> 3 días), estuvo asociada significativamente a una evolución adversa con fallecimiento o alteraciones neurológicas al alta ( $p=0,01$ prueba bilateral de Fisher) (Tabla 3 ). Las alteraciones neurológicas al alta no pudieron ser clasificadas debido al diseño retrospectivo del trabajo y a la variedad de descripciones clínicas no sistematizadas sobre este compromiso. No se observó una asociación entre la edad del paciente y la presencia de compromiso de conciencia al ingreso.

Excluyendo un paciente que fue trasladado a otro centro a los 4 días de tratamiento, el promedio de hospitalización fue de 21,4 días (rango 5 a 64 días, DS 16,7). Los extremos corresponden a los dos pacientes que fallecieron en la serie.

\section{Discusión}

De acuerdo a nuestro conocimiento, esta serie representa la publicación con el mayor número de casos reportados de encefalitis herpética en nuestro país y la única serie en pacientes adul$\operatorname{tos}^{4-7}$. La búsqueda combinada en dos hospitales universitarios permitió identificar 15 casos para una enfermedad de presentación esporádica. La probabilidad de haber incluido erróneamente un caso es muy baja por la alta especificidad del examen utilizado como criterio de inclusión ${ }^{10}$.

Distribución anual, estacional y etaria. La incidencia anual de encefalitis no es homogénea y nuestros resultados, con una variación anual de casos, son concordantes con esta descripción. La incidencia de encefalitis herpética en una población varía significativamente y puede llegar a duplicarse en diferentes décadas ${ }^{11}$. El predominio otoñal de casos observado en nuestra serie también ha sido reportado. La encefalitis herpética es una infección que afecta predominantemente a adultos mayores de 30 años tal como fue observado en esta serie, aunque un tercio de los casos se presenta antes de esa edad ${ }^{2}$. Ambos hospitales tienen un bajo número de camas pediátricas y eso explica el fuerte predominio de casos adultos en nuestra serie.

Presentación clínica y alteraciones del LCR. Las manifestaciones clínicas de la encefalitis herpética son inespecíficas, aunque en ocasiones los pacientes presentan una constelación de signos fronto-temporales como afasia, cambios de personalidad y convulsiones focales que sugieren el diagnóstico. La presencia de compromiso de conciencia, fiebre, cefalea, cambios de personalidad, convulsiones, vómitos, hemiparesia o pérdida de memoria ocurren con la misma frecuencia que las observadas en otras causas de encefalitis $^{12}$. Aunque en nuestra serie la presencia de manifestaciones herpéticas cutáneas o mucosas fue infrecuente, hasta un tercio de los pacientes puede presentarse con este fenómeno.

Los resultados de este estudio confirman la presentación habitual de este cuadro, su rápida progresión expresada en la corta duración de los síntomas antes del ingreso y la pleocitosis de predominio mononuclear con euglucorraquia y discreto aumento de las proteínas en el LCR. Aunque $60 \%$ de nuestros pacientes presentaron eritrocitos en el LCR, la detección de ellos no ha constituido un elemento diferencial en grandes series de pacientes ${ }^{12}$. De la misma manera, la pleocitosis no constituye un hallazgo específico siendo encontrada en otras causas de encefalitis. 
Aunque inespecífico, cifras superiores a 50 células $/ \mathrm{mm}^{3}$ están significativamente asociadas a una encefalitis herpética ${ }^{12}$.

Diagnóstico diferencial. Aunque no evaluado en nuestro trabajo, el diagnóstico diferencial de la encefalitis herpética es amplio y merece una discusión especial. La primera distinción es separar un cuadro de encefalopatía de uno de encefalitis. A favor de este último, se asocian fiebre, cefalea, signos neurológicos focales, convulsiones generalizadas o focales, leucocitosis, pleocitosis, enlentecimiento difuso o alteraciones focales en el EEG y anormalidades focales en la $\mathrm{RM}^{13}$. Las infecciones sistémicas o encefalopatías metabólicas asociadas a cuadros febriles secundarios constituyen un escenario común donde debe ser diferenciados ambos tipos de patologías.

La segunda tarea es diferenciar una encefalitis por VHS de otras causas infecciosas o postinfecciosas tales como las asociadas al virus varicela-zoster, citomegalovirus, virus de EpsteinBarr, encefalitis por adenovirus, influenza A, parotiditis, rabia, arbovirus o enterovirus. Las encefalomielitis postinfecciosas se presentan habitualmente hasta cuatro semanas después de una inmunización o infección natural por sarampión, rubéola o varicela o influenza. Una serie de elementos clínicos, epidemiológicos y de laboratorio permiten este diagnóstico diferencial. El lector interesado puede consultar revisiones sobre el tema ${ }^{13}$. Baste por ahora señalar que en nuestro país, las principales causas corresponden a VHS y enterovirus.

Estudio con EEG, TAC y RM. Las alteraciones localizadas pesquisadas mediante diferentes procedimientos de apoyo, son observadas con una frecuencia significativamente superior en pacientes con encefalitis herpética en comparación a otras causas y, en un contexto clínico apropiado, apoyan el diagnóstico. Esto es particularmente útil en la distinción entre encefalitis y encefalopatía ${ }^{13}$. Sin embargo, su sensibilidad y especificidad no son óptimas. Considerando estudios con un estándar de oro apropiado y grandes series de pacientes, la EEG aparece más sensible que la TAC cerebral para estos propósitos. Por ejemplo, en una serie de 113 pacientes con diagnóstico confirmado por biopsia cerebral, $81 \%$ presentó un EEG alterado de tipo focal versus $59 \%$ de pacientes con alteraciones en la $\mathrm{TAC}^{12}$. Sin embargo, otras patologías tales como abscesos, encefalitis por virus de Epstein-Barr, tumores o tuberculosis o infecciones por Cryptococcus neoformans o Toxoplasma gondii, tam- bién presentan cambios EEG o en la TAC cerebral con una frecuencia elevada (59 y $22 \%$, respectivamente) lo que restringe la especificidad de estos dos exámenes ${ }^{12}$.

Las alteraciones sugerentes en un EEG corresponden a lentitud del ritmo en la zona temporal, actividad epileptiforme en la misma zona y la aparición de PLEDS, esta última considerada de alta especificidad ${ }^{13,14}$. En nuestra serie, la alteración más frecuente correspondió a la actividad epileptiforme temporal, presente en casi la mitad de los casos en los cuales se hizo un EEG.

La RM tiene un valor indiscutido en el estudio de pacientes con manifestaciones neurológicas centrales y es actualmente considerado el método por imágenes de elección ante casos sospechosos de encefalitis ${ }^{13}$. Sin embargo, su capacidad para reconocer cuadros específicos de encefalitis herpética es imperfecta, debido a la todavía insuficiente descripción de sus alteraciones, a la aplicación de ella en diferentes momentos evolutivos de la enfermedad y las diferentes metodologías de esta técnica que han ido variando en los últimos años, por ejemplo con la incorporación de estudios FLAIR, de difusión o de espectrometría ${ }^{15}$. Estas limitaciones han impedido conocer con exactitud su sensibilidad y especificidad. En una pequeña serie de casos confirmados por RPC, la RM cerebral mostró alteraciones focales en el lóbulo temporal en aproximadamente $80 \%$ de los pacientes; sin embargo, lesiones similares fueron observadas en $12,5 \%$ de pacientes con una prueba de RPC negativa asociadas a abscesos bacterianos, encefalomielitis aguda diseminada, encefalitis del tronco cerebral o encefalitis por anticuerpos monoclonales ${ }^{16}$. De esta manera, la RM ofrecería una sensibilidad superior a la TAC, similar o inferior al EEG pero con una especificidad superior a cualquiera de ellas dos. Por esta razón, la RM ha desplazado al EEG y a la TAC como el procedimiento de elección para el estudio neurodiagnóstico ante la sospecha de encefalitis herpética. Durante los primeros días de evolución, el EEG y la RM ofrecen la mejor sensibilidad diagnóstica. En nuestro estudio se observó, al igual que lo reportado en la literatura, una mejor sensibilidad para EEG $(91,7 \%)$, superior a la obtenida por RM $(81,8 \%)$. La RM permite detectar anormalidades que reflejan una encefalitis necrosante que afecta principalmente las superficies orbitales del lóbulo frontal y la porción infero-medial del lóbulo temporal incluyendo el sistema límbico. Las lesiones son habitualmente hipointensas en la señal T1 e hiperintensas en $\mathrm{T} 2^{16}$.

Los hallazgos sugerentes en la TAC cerebral 
corresponden a edema localizado, lesiones hipodensas, efecto de masa, hemorragia y/o refuerzo con medio de contraste con un patrón anatómico similar a la RM. Estos cambios son menos frecuentes en la primera semana de evolución ${ }^{11,12,16}$. Aunque la cifra de sensibilidad para la TAC en nuestra serie fue notoriamente inferior a lo habitualmente descrito ( $13 \%$ versus $\sim 50-66 \%$ ), esta diferencia es probablemente explicada por la precocidad del examen. En nuestra serie, la TAC fue realizada durante los primeros 7 días de evolución en la mayor parte de los casos (73\%). Sin embargo, la RM permitió una mejor utilidad diagnóstica para la misma ventana de tiempo, aunque fue aplicada en una mayor proporción antes de la primera semana $(90 \%)$. Actualmente se recomienda el uso de la TAC cerebral como un procedimiento alternativo cuando no hay disponibilidad de $\mathrm{RM}^{13}$. Se desconoce el valor de TAC sucesivas en este contexto; sin embargo, el advenimiento de la RM y el estudio del LCR con RPC hacen poco probables mayores estudios sobre este tópico.

Diagnóstico microbiológico. El diagnóstico de certeza de la encefalitis herpética se puede establecer mediante inmunofluorescencia, microscopia electrónica, cultivo viral de una biopsia cerebral de tejido temporal o mediante la demostración de síntesis intratecal de anticuerpos anti virus herpes simplex ${ }^{3,6}$. Sin embargo, estas estrategias son laboriosas o invasoras y han sido reemplazada por la detección del virus tipo 1 ó 2 mediante RPC en una muestra de LCR. Al igual que en nuestra serie, la mayor parte de los casos en adultos están asociados al tipo 1. El estudio de VHS por RPC se introdujo progresivamente desde el año $1990^{5,17,18}$. Este examen se efectúa en laboratorios especializados y la muestra puede conservarse varios días refrigerada a $4^{\circ} \mathrm{C}$. El volumen de la muestra requerido es mínimo (1 $\mu \mathrm{L})$ y estrictamente sólo se requieren $100 \mu \mathrm{L}$ $(0,1 \mu \mathrm{L})$. La positividad de este examen se extiende hasta los 25 días desde el inicio de la encefalitis y hasta los 7 días desde el inicio de un tratamiento con aciclovir. Esta estrategia es de alto rendimiento con una sensibilidad de $96-98 \%$ y una especificidad de $94-99 \%$, superior a los magros resultados asociados al cultivo viral $(\leq 30 \% \text { sensibilidad })^{10}$.

\section{Tratamiento y evolución}

A pesar de los avances en eficacia obtenidos con aciclovir, el tratamiento de la encefalitis herpética se caracteriza por una letalidad significativa que bordea el $20 \%$, por el desarrollo de secuelas graves en aproximadamente 50\% de los sobrevivientes y finalmente, por el riesgo de recaída. La cifra de letalidad y de secuelas al momento de egreso fue similar en nuestra serie a lo reportado en la literatura ${ }^{2}$.

El tratamiento de referencia de la encefalitis por VHS es aciclovir luego de que un estudio randomizado publicado en 1986 demostrara la superioridad de este compuesto sobre vidarabina, el antiviral de referencia hasta entonces. La letalidad en el grupo tratado con aciclovir fue de $19 \%$ los 6 meses versus $54 \%$ con vidarabina ${ }^{2}$.

El pronóstico de esta infección depende de la precocidad del tratamiento (casi $0 \%$ de letalidad si se inicia en los primeros 4 días), de la edad del paciente (empeora en pacientes mayores de 30 años y se concentra especialmente en los mayores de 60 años) y de la severidad inicial del cuadro (con puntaje Glasgow $\leq 6$ la letalidad a los 6 meses alcanza a $25 \%$ en contraste al $0 \%$ cuando el puntaje es superior a 10) ${ }^{2,19}$. En algunas series retrospectivas, el diagnóstico es sospechado al ingreso en una minoría de pacientes (38\%) siendo la causa más frecuente de diagnóstico erróneo, la postergación de una punción lumbar por la asunción de una encefalopatía tóxicometabólica atribuida a una infección sistémica ${ }^{19}$. La baja sospecha diagnóstica explica el retraso del tratamiento (en algunas series hasta 18 días), un factor determinante para el pronóstico de esta enfermedad. En nuestra serie observamos que el inicio del tratamiento en un plazo superior a los 3 días desde el inicio de los síntomas estuvo significativamente asociado a la muerte del paciente o a la presencia de alteraciones neurológicas al momento del egreso.

La causa de muerte en uno de los dos pacientes fallecidos por encefalitis, afectado por SIDA con alta carga viral y un linfoma tipo Burkitt del seno cavernoso, no pudo ser atribuida directamente a la infección herpética. Sólo $2 \%$ de los pacientes con infección por VIH y síntomas neurológicos tienen una infección herpética del SNC. Tal como fue observado en nuestros resultados, la encefalitis herpética no es una enfermedad asociada a inmunodepresión. Sin embargo, en pacientes con infección por VIH y muy bajo recuento de linfocitos $\mathrm{CD} 4$, la infección es subaguda, con una baja tasa de respuesta y alto riesgo de recaída ${ }^{20}$.

De acuerdo a las recomendaciones actuales, la dosis de terapéuticas de aciclovir deben ser: 10 $\mathrm{mg} / \mathrm{kg} /$ dosis cada 8 horas (30 mg/kg/día) aplicadas por vía endovenosa, durante 14 a 21 días $^{14}$. 
Ello porque se ha observado un riesgo de recurrencia cuando el tratamiento no alcanza a los 14 días o cuando la dosis total acumulada es baja $(<300 \mathrm{mg} / \mathrm{kg})^{21}$. La mayor parte de las recaídas son observadas en las dos semanas siguientes al término del tratamiento, se presentan con un cuadro clínico similar y en general tienen una RPC negativa para VHS ${ }^{21}$.

La administración endovenosa de este compuesto asegura concentraciones plasmáticas post infusión de $20 \mathrm{mg} / \mathrm{ml}$, superior a la concentración inhibitoria en cultivos celulares para VHS 1 y 2 . ( $\leq 0,9 \mathrm{mg} / \mathrm{ml} \mathrm{y} \leq 2,2 \mathrm{mg} / \mathrm{ml}$, respectivamente). La concentración en el LCR es aproximadamente la mitad de la concentración observada en el plasma. La baja biodisponibilidad de aciclovir oral impide recomendar esta formulación para esta enfermedad; la concentración máxima obtenida por vía oral con altas dosis de aciclovir alcanza $1,6 \mathrm{mg} / \mathrm{ml}$ en el plasma y cifras inferiores en el LCR ${ }^{22}$. Hasta la fecha no se han publicado ensayos clínicos desarrollados con valaciclovir, un fármaco de uso oral, con mayor biodisponibilidad que aciclovir.

El pronóstico y las secuelas neurológicas asociadas a esta enfermedad después del egreso hospitalario no son favorables. En un estudio de seguimiento, McGrath y cols observaron una mortalidad post egreso concentrada en los primeros dos años post alta y que casi igualaba la mortalidad observada en el hospital ${ }^{19}$. Sólo aproximadamente $48 \%$ logró una recuperación adecuada para continuar en forma autónoma con las actividades de la vida diaria y lograr una reinserción laboral o rendimiento escolar similar al desarrollado antes de la encefalitis. Otro $21 \%$ terminó con una discapacidad moderada, con posibilidades de una vida autónoma pero con dificultades para encontrar trabajo o con grandes limitaciones escolares ${ }^{19}$. Los pacientes sobrevivientes pueden presentar como secuelas convulsiones, problemas de memoria u orientación, hemianopsia, afasia, cambios de personalidad mayores o depresión. En grados extremos, un estado vegetativo persistente. Aunque el diseño de nuestro trabajo impide conocer la evolución de las secuelas que estaban presentes al egreso, la cifra observada es concordante con otras publicaciones $^{2}$.

Nuestra serie clínica presenta varias limitaciones o sesgos. Entre ellos la baja representación de la población infantil, las limitaciones para conocer la evolución post egreso, la frecuencia de recaídas o las secuelas a largo plazo. Tampoco permite estudiar la especificidad de las alteraciones en el EEG, TAC o RM cerebral. No obstante, permite dar cuenta del enfoque y manejo actual de esta enfermedad en nuestro país, de la importancia de la sospecha precoz y de los resultados obtenidos con el tratamiento.

\section{Resumen}

La encefalitis herpética es la causa más frecuente de encefalitis esporádica en el mundo occidental. Para conocer las principales características clínicas de esta enfermedad en nuestro medio, se efectuó un análisis de casos confirmados por reacción de polimerasa en cadena en dos hospitales universitarios de Santiago. Un total de 15 casos pudo ser identificado con un promedio de edad de 41 años (578 años) y $80 \% \geq 30$ años, el primero de ellos el año 1998. La mayor parte se presentó con fiebre y compromiso de conciencia ( $80 \%$ cada uno) o cefalea $(67 \%)$. Las convulsiones y la focalización fueron infrecuentes $(\leq 15 \%)$ y sólo 2 casos $(13 \%)$ tuvieron además una manifestación herpética extracerebral. La duración promedio de los síntomas fue de 3,8 días. La mayor parte estuvo asociada al serotipo 1 $(86,7 \%)$. El 91,7\% de los casos evaluados presentó alteraciones electroencefalográficas, $81,8 \%$ alteraciones en la resonancia magnética y sólo 13,3\% en la tomografía axial computarizada. La totalidad de los pacientes fue tratada con aciclovir y la letalidad fue de $13,3 \%$, aunque el deceso en un paciente con SIDA y linfoma del SNC no pudo ser atribuido a la infección herpética. Seis pacientes (40\%) presentaban secuelas neurológicas al momento del egreso. La muerte o alteraciones neurológicas al alta estuvieron asociadas significativamente a un inicio del tratamiento $>3$ días desde el inicio de los síntomas. ( $\mathrm{p}=0,01$ prueba bilateral de Fisher).

Agradecimientos. Los autores desean agradecer los valiosos aportes de Mario Díaz S, Neurólogo del Hospital Clínico de la Universidad de Chile y a Mónica Peña C, Tecnólogo Médico del Programa de Virología de la Facultad de Medicina de la Universidad de Chile.

\section{Bibliografía}

1.- Whitley R J, Gnann J W. Viral encephalitis: familiar infections and emerging pathogens. Lancet 2002; 359: 507-14.

2.- Whitley R J, Alford C, Hirsch M S, Schooley R T, Luby J P, Aoki F Y, et al. Vidarabine versus aciclovir therapy in herpes simplex encephalitis. N Eng J Med 1986; 314: 144-9.

3.- Jara J, Burgos R, Ríos R, Benítez H, Mercado M. Comunicación caso clínico de encefalitis herpética demostrada por biopsia cerebral con microscopia electrónica. Rev Chil Infect 1988; 5: 61-6.

4.- Ruiz-Esquide F, Peña M, Pineur E, Henríquez M T, Hernández A, Larrañaga C. Encefalitis herpética neonatal. Caso clínico y revisión del tema. Rev Chil Pediatr 2002; 73: 159-63. 
5.- Schultz R, Larrañaga C, Wu E, Suárez M. Infección congénita por virus herpes simplex. Rev Chil Pediatr 1991; 62: 44-7.

6.- Wu E, Grado C. Infecciones del sistema nervioso central por virus herpes simplex. Rev Chil Infect 1985; 2: 83-92.

7.- Wu E, Jerez E, Muñoz G, Cabrera F. Casos de encefalitis herpética con aislamiento viral en líquido cefalorraquídeo. Rev Chil Infect 1985; 2: 132-7.

8.- Schlesinger Y, Buller R S, Brunstrom J E, Moran C J, Storch G A. Expanded spectrum of herpes simplex encephalitis in childhood. J Pediatr 1995; 126: 234-41.

9.- Casas I, Tenorio A, Echevarria J M, Klapper P E, Cleator G M. Detection of enteroviral RNA and specific DNA of herpesviruses by multiplex genome amplification. J Virol Methods 1997; 66: 39-50.

10.- Lakeman F D, Whitley R J, National Institute of Allergy and Infectious Diseases Collaborative Antiviral Study Group. Diagnosis of herpes simplex encephalitis: application of polymerase chain reaction to cerebrospinal fluid from brain-biopsied patients and correlation with disease. J Infect Dis 1995; 171: 857-63.

11.- Rantalaiho T, Farkkila M, Vaheri A, Koskiniemi M. Acute encephalitis from 1967 to 1991 . J Neurol Sci 2001; 184: 169-77.

12.- Whitley R J, Soong S J, Linneman C, Liu C, Pazin G, Alford C A, et al. Herpes simplex encephalitis. Clinical assessment. JAMA 1982; 247: 317-20.

13.- Kennedy P G E. Viral encephalitis: causes, differential diagnosis, and management. J Neurol Neurosurg Psychiatry 2004; 75 (Suppl I): i10-i15.

14.- Tyler K L. Herpes simplex virus infections of the central nervous system: encephalitis and meningitis, including Mollaret's. Herpes 2004; 11 (Suppl 2): 57A-64A.
15.- Maschke M, Kastrup O, Forsting M, Diener H C. Update on neuroimaging in infectious central nervous system disease. Curr Opin Neurol 2004; 17: 475-80.

16.- Domingues R B, Fink M C D, Tsanaclis A M C, de Castro C C, Cerri G G, Mayo M S, et al. Diagnosis of herpes simplex encephalitis by magnetic resonance imaging and polymerase chain reaction assay of cerebrospinal fluid. J Neurol Sci 1998; 157: 148-53.

17.- Powell K F, Anderson N E, Frith R W, Coroxson M C. Non-invasive diagnosis of herpes simplex encephalitis. Lancet 1990; 335: 357-8.

18.- Rowley A H, Whitley R J, Lakeman F D, Wolinsky S M. Rapid detection of herpes-simplex-virus DNA in cerebrospinal fluid of patients with herpes simplex encephalitis. Lancet 1990; 335: 440-1.

19.- McGrath N, Anderson N E, Croxson M C, Powell K F. Herpes simplex encephalitis treated with acyclovir: diagnosis and long-term outcome. J Neurol Neurosurg Psychiatry 1997; 63: 321-6.

20.- Cinque P, Vago L, Marenzi R, Giudici B, Weber T, Corradini R, et al. Herpes simplex virus infections of the central nervous system in human immunodeficiency virus-infected patients: Clinical management by polymerase chain reaction assay of cerebrospinal fluid. Clin Infect Dis 1998; 27: 303-9.

21.- Ito Y, Kimura H, Yabuta Y, Ando Y, Murakami T, Shiomi A, et al. Exacerbation of herpes simplex encephalitis after successful treatment with acyclovir. Clin Infect Dis 2000; 30: 185-7.

22.- Hayden FG. Antiviral drugs (other than antiretrovirals). En Mandell, Douglas and Bennett's Principles and Practice of infectious Diseases. Mandell GL, Bennett JE, Dolin R (eds). (5 ${ }^{\text {th }}$ edition). Churchill Livingstone New York 2000 pp 460-91.

Correspondencia a:

Alberto Fica Cubillos

afica@ns.hospital.uchile.cl 